\title{
Isocurvature perturbations in dark radiation
}

\author{
Etsuko Kawakami \\ Institute for Cosmic Ray Research, University of Tokyo, Kashiwa 277-8582, Japan \\ E-mail: kwkm@icrr.u-tokyo.ac.jp \\ Masahiro Kawasaki \\ Institute for Cosmic Ray Research, University of Tokyo, Kashiwa 277-8582, Japan \\ Institute for the Physics and Mathematics of the Universe, University of Tokyo, Kashiwa \\ 277-8568, Japan \\ E-mail: kawasakieicrr.u-tokyo.ac.jp
}

\section{KOICHI MIYAMOTO*}

Institute for Cosmic Ray Research, University of Tokyo, Kashiwa 277-8582, Japan

E-mail: miyamonedicrr.u-tokyo.ac.jp

\section{Kazunori Nakayama}

Department of Physics, University of Tokyo, Bunkyo-ku, Tokyo 113-0033, Japan

E-mail. kazunori@hep-th.phys.s.u-tokyo.ac.jp

\section{Toyokazu Sekiguchi}

Department of Physics and Astrophysics, Nagoya University, Nagoya 464-8602, Japan E-mail.toyokazu.sekiguchi@nagoya-u.jp

\begin{abstract}
Recent cosmological observations such as measurements of the CMB anisotropy indicate the existence of non-interacting relativistic particles, dubbed the "extra radiation" in the Universe beyond the standard three neutrino species. In this paper we explore the possibility that the dark radiation, the mixture of the extra radiation and neutrino, has isocurvatrue fluctuations. A general formalism to evaluate isocurvature perturbations in the dark radiation is provided in the mixed inflaton-curvaton system, where the dark radiation is produced by the decay of both scalar fields. We also derive constraints on the abundance of the dark radiation and the amount of its isocurvature perturbation. These constraints are applied to some particle physics motivated models. Besides, we discuss the non-Gaussianity of the dark radiation isocurvature perturbation and forecast the constraint on it in the future CMB experiments.
\end{abstract}

VIII International Workshop on the Dark Side of the Universe

June 10-15, 2012

B? zios, Rio de Janeiro, Brasil

\footnotetext{
${ }^{*}$ Speaker.
} 


\section{Introduction}

Some recent experiments suggest that the effective number of neutrino species $N_{\text {eff }}$ might be larger than $3.046{ }^{1}$ by $\Delta N_{\text {eff }} \approx 1$. For example, the constraint from the WMAP measurement of the cosmic microwave background (CMB) anisotropy combined with observations of the baryon acoustic oscillation (BAO) and the Hubble parameter (H0) is $N_{\text {eff }}=4.34_{-0.88}^{+0.86}$ (68\%C.L.) [1]. Such results imply that there might be non-interacting energy component other than neutrino. Hereafter we call such a fluid "extra radiation" and the mixture of it and neutrino "dark radiation"(DR).

There are also theoretical motivations to consider the extra radiation in the particle physics beyond the Standard Model. For example, axion, a light scalar filed introduced to solve the strong CP problem, is a good candidate of the extra radiation. In theoretical papers treating the extra radiation, it has been so far (implicitly) assumed that the extra radiation has the adiabatic perturbation. However, the property of the fluctuation of the extra radiation depends on its production mechanism. For example, there are scenarios that the decay of a scalar field nonthermally produces the extra radiation. Axion produced by decay of saxion, the scalar partner of axion in the supersymmetric axion models, is among such scenarios. In such a case, it succeeds the fluctuation of the source scalar field, which arises as a quantum fluctuation during inflation. Therefore, the extra radiation can have an independent perturbation from the adiabatic one, an isocurvature perturbation. Investigating the isocurvature perturbation in the extra radiation may have a potential to distinguish the model of the extra radiation, if future observations further confirm $\Delta N_{\text {eff }} \approx 1$.

Therefore, in this paper we derive constraints on the extra radiation from the currently available datasets, considering the effect of the extra radiation. In addition to its isocurvature perturbation, which affects the $\mathrm{CMB}$ anisotropy similarly to the neutrino isocurvature perturbation, its energy density itself affects the CMB through the change of the Hubble expansion rate. Due to these effects, we can constrain $N_{\text {eff }}$ and the amplitude of the isocurvature perturbation in the DR $S_{D R}$ using the CMB data. Besides, the DR isocurvature perturbation can have large non-Gaussianities in some cases. We consider how strongly such non-Gaussianities will be constrained by future CMB experiments. As a theoretical model of the extra radiation, we focus on the scenario that it is produced by decay of inflaton or the other scalar field or both. We derive the formulas for $N_{\text {eff }}, S_{D R}$ and non-Gaussianities and consider the implication of observational constraints for that model.

\section{The model and the formalism}

We consider a cosmological model with two scalar fields: the inflaton $\phi$ and the "curvaton" $\sigma$, which is light during inflation and decays later than inflaton. They obtain large-scale quantum fluctuations and may generate isocurvature perturbations as well as adiabatic ones.

All the components of the current Universe, photon (denoted by $\gamma$ ), neutrino (denoted by $v$ ), dark matter, baryon and extra radiation (denoted by $X$ ) arise from both $\phi$ and $\sigma$. The temporal change of the components of the Universe is summarized in Table 1. Here, we write the Standard Model radiation (plus dark matter) which exists prior to the neutrino decoupling as $r$. This model can be characterized by the three parameters: the branching ratio of inflaton decay into $r r_{\phi}$, that of curvaton $r_{\sigma}$ and the ratio of the curvaton energy density to the total energy density at its decay $R_{\sigma}$.

\footnotetext{
${ }^{1}$ The standard value of $N_{\text {eff }}$ is not 3 but $\simeq 3.046$, due to partial heating of the neutrinos at $e^{ \pm}$annihilation.
} 
Table 1: Energy components of the Universe at each epoch except for CDM and baryon. Here $\Gamma_{\phi}\left(\Gamma_{\sigma}\right)$ is the decay rate of the inflaton (curvaton), and $\Gamma_{v}$ denotes the neutrino interaction rate at the neutrino freezeout. $\Gamma_{e^{ \pm}}$denotes the Hubble parameter at the $e^{ \pm}$annihilation. $r_{e}$ denotes the plasma consisting of $\gamma$ and $e^{ \pm}$. The superscripts $(\phi)$ and $(\sigma)$ represent the origin of the fluid.

\begin{tabular}{|c|c|c|}
\hline epoch & component & energy transfer \\
\hline \hline$\Gamma_{\phi}<H$ & $\phi, \sigma$ & $\phi \rightarrow X^{(\phi)}+r^{(\phi)}$ \\
\hline$\Gamma_{\sigma}<H<\Gamma_{\phi}$ & $X^{(\phi)}, r^{(\phi)}, \sigma$ & $\sigma \rightarrow X^{(\sigma)}+r^{(\sigma)}$ \\
\hline$\Gamma_{\mathrm{v}}<H<\Gamma_{\sigma}$ & $X, r$ & $r \rightarrow \mathrm{v}+r_{e}$ \\
\hline$\Gamma_{e^{ \pm}}<H<\Gamma_{\mathrm{v}}$ & $X, v, r_{e}(\mathrm{DR}=X+\mathrm{v})$ & $e^{ \pm} \rightarrow \gamma$ \\
\hline$H<\Gamma_{e^{ \pm}}$ & $X, v, \gamma(\mathrm{DR}=X+v)$ & \\
\hline
\end{tabular}

We define the adiabatic perturbation $\zeta$ as the curvature perturbation on the uniform density slice. The isocurvature perturbation of DR is defined as

$$
S_{D R} \equiv 3\left(\zeta_{D R}-\zeta\right)
$$

Here the curvature perturbation of DR, $\zeta_{D R}$ is defined as

$$
\rho_{D R}(\vec{x})=\bar{\rho}_{i} e^{4\left(\zeta_{D R}-\zeta\right)}
$$

where $\rho_{D R}(\vec{x})$ is evaluated on the uniform density slice. $\zeta$ and $S_{D R}$ are determined by the quantum fluctuation of scalar fields, $\delta \phi$ and $\delta \sigma$. Up to the second order of them, $\zeta$ and $S_{D R}$ are expanded as

$$
\zeta=\sum_{i} N_{\phi_{i}} \delta \phi_{i}+\sum_{i, j} \frac{1}{2} N_{\phi_{i} \phi_{j}} \delta \phi_{i} \delta \phi_{j}, S_{D R}=\sum_{i} S_{\phi_{i}} \delta \phi_{i}+\sum_{i, j} \frac{1}{2} S_{\phi_{i} \phi_{j}} \delta \phi_{i} \delta \phi_{j}
$$

where $\delta \phi_{i}$ is either $\delta \phi$ or $\delta \sigma$. Up to the leading term, the power spectra of $\zeta$ and $S_{D R}$ are given by

$$
\left\langle A_{1}\left(\vec{k}_{1}\right) A_{2}\left(\vec{k}_{2}\right)\right\rangle \equiv(2 \pi)^{3} \delta\left(\vec{k}_{1}+\vec{k}_{2}\right) P_{A_{1} A_{2}}\left(k_{1}\right),
$$

where $A_{i}$ denotes either $\zeta$ or $S_{D R}$ and

$$
P_{\zeta \zeta}(k)=\left[N_{\phi}^{2}+N_{\sigma}^{2}\right] P_{\delta \phi}(k), P_{\zeta S_{\mathrm{DR}}}(k)=\left[N_{\phi} S_{\phi}+N_{\sigma} S_{\sigma}\right] P_{\delta \phi}(k), P_{S_{\mathrm{DR}} S_{\mathrm{DR}}}(k)=\left[S_{\phi}^{2}+S_{\sigma}^{2}\right] P_{\delta \phi}(k),
$$

The power spectrum of $\delta \phi_{i}(\delta \phi$ or $\delta \sigma)$ is defined as

$$
\begin{aligned}
\left\langle\delta \phi_{i}\left(\vec{k}_{1}\right) \delta \phi_{j}\left(\vec{k}_{2}\right)\right\rangle & \equiv(2 \pi)^{3} \delta\left(\vec{k}_{1}+\vec{k}_{2}\right) P_{\delta \phi}\left(k_{1}\right) \delta_{i j}, \\
P_{\delta \phi}(k) & =\frac{H_{\mathrm{inf}}^{2}}{2 k^{3}}\left(\frac{k}{k_{0}}\right)^{n_{s}-1},
\end{aligned}
$$

where $H_{\text {inf }}$ is the Hubble parameter during inflation, $n_{s}$ is the scalar spectral index ${ }^{2}$ and $k_{0}$ is the pivot scale chosen as $k_{0}=0.002 \mathrm{Mpc}^{-1}$. The amplitude of the isocurvature perturbation and the correlation between it and the adiabatic one are parametrized by $\alpha$ and $\gamma$, which are defined as

$$
\left(\begin{array}{cc}
\mathscr{P}_{\zeta \zeta}(k) & \mathscr{P}_{\zeta S_{\mathrm{DR}}}(k) \\
\mathscr{P}_{\mathrm{D}_{\mathrm{DR}} \zeta}(k) & \mathscr{P}_{S_{\mathrm{DR}} S_{\mathrm{DR}}}(k)
\end{array}\right)=A_{s}\left(\frac{k}{k_{0}}\right)^{n_{s}-1}\left(\begin{array}{cc}
1-\alpha & \gamma \sqrt{\alpha(1-\alpha)} \\
\gamma \sqrt{\alpha(1-\alpha)} & \alpha
\end{array}\right),
$$

\footnotetext{
${ }^{2}$ The scalar spectral indices for $\phi$ and $\sigma$ do not coincide in general. In the following we assume they are the same just for simplicity.
} 
where $\mathscr{P}_{A B}(k) \equiv\left(k^{3} / 2 \pi^{2}\right) P_{A B}(k)$ and $A_{s} \approx 2 \times 10^{-9}$ is the amplitude of primordial power spectra. $\gamma=0, \gamma=+1$ and $\gamma=-1$ mean that the adiabatic perturbation and the DR isocurvature perturbation are uncorrelated, totally correlated and totally anti-correlated, respectively. The bispectra of $\zeta$ and $S_{D R}$ are defined as

$$
\left\langle A_{1}\left(\vec{k}_{1}\right) A_{2}\left(\vec{k}_{2}\right) A_{3}\left(\vec{k}_{3}\right)\right\rangle \equiv(2 \pi)^{3} \delta\left(\vec{k}_{1}+\vec{k}_{2}+\vec{k}_{3}\right) B_{A_{1} A_{2} A_{3}}\left(k_{1}, k_{2}, k_{3}\right) .
$$

We define the non-Gaussianity parameters $f_{N L}^{A_{1}, A_{2} A_{3}}$, which represent magnitudes of non-Gaussianities, as

$$
B^{A_{1} A_{2} A_{3}}\left(k_{1}, k_{2}, k_{3}\right)=f_{N L}^{A_{1}, A_{2} A_{3}}\left(k_{1}, k_{2}, k_{3}\right) P_{\zeta \zeta}\left(k_{2}\right) P_{\zeta \zeta}\left(k_{3}\right)+(2 \text { cyclics of }\{123\}),
$$

They are written in terms of the coefficients in (2.3) as

$$
\begin{array}{r}
f_{\mathrm{NL}}^{\zeta, \zeta \zeta} \equiv f_{N L}^{(1)}=\frac{\sum_{i, j} N_{\phi_{i}} N_{\phi_{j}} N_{\phi_{i} \phi_{j}}}{\left(\sum_{i} N_{\phi_{i}}^{2}\right)^{2}}, f_{\mathrm{NL}}^{S_{\mathrm{DR}}, \zeta \zeta} \equiv f_{N L}^{(2)}=\frac{\sum_{i, j} S_{\phi_{i}} N_{\phi_{j}} N_{\phi_{i} \phi_{j}}}{\left(\sum_{i} N_{\phi_{i}}^{2}\right)^{2}}, \\
f_{\mathrm{NL}}^{\zeta, S_{\mathrm{DR}} \zeta}=f_{\mathrm{NL}}^{\zeta, \zeta S_{\mathrm{DR}}} \equiv f_{N L}^{(3)}=\frac{\sum_{i, j} N_{\phi_{i}} N_{\phi_{j}} S_{\phi_{i} \phi_{j}}}{\left(\sum_{i} N_{\phi_{i}}^{2}\right)^{2}}, f_{\mathrm{NL}}^{\zeta, S_{\mathrm{DR}} S_{\mathrm{DR}}} \equiv f_{N L}^{(4)}=\frac{\sum_{i, j} N_{\phi_{i}} S_{\phi_{j}} S_{\phi_{i} \phi_{j}}}{\left(\sum_{i} N_{\phi_{i}}^{2}\right)^{2}} \\
f_{\mathrm{NL}}^{S_{\mathrm{DR}}, \zeta S_{\mathrm{DR}}}=f_{\mathrm{NL}}^{S_{\mathrm{DR}}, S_{\mathrm{DR}} \zeta} \equiv f_{N L}^{(5)}=\frac{\sum_{i, j} S_{\phi_{i}} S_{\phi_{j}} N_{\phi_{i} \phi_{j}}}{\left(\sum_{i} N_{\phi_{i}}^{2}\right)^{2}}, f_{\mathrm{NL}}^{S_{\mathrm{DR}}, S_{\mathrm{DR}} S_{\mathrm{DR}}} \equiv f_{N L}^{(6)}=\frac{\sum_{i, j} S_{\phi_{i}} S_{\phi_{j}} S_{\phi_{i} \phi_{j}}}{\left(\sum_{i} N_{\phi_{i}}^{2}\right)^{2}}
\end{array}
$$

up to the leading order ${ }^{3}$.

In the model considered in this paper, we can calculate the coefficients in (2.3) using $\delta N$ formalism[4] 5] as [2],

$$
\begin{gathered}
N_{\phi}=\frac{1}{M_{\mathrm{P}}^{2}} \frac{V}{V_{\phi}}, N_{\phi \phi}=\frac{1}{M_{\mathrm{P}}^{2}}\left(1-\frac{V V_{\phi \phi}}{V_{\phi}^{2}}\right), N_{\sigma}=\frac{3+R}{6 \sigma_{i}}\left(\frac{\hat{R}_{r} R_{r}^{(\sigma)}}{R_{r}}+\frac{\hat{R}_{X} R_{X}^{(\sigma)}}{R_{X}}\right), \\
N_{\sigma \sigma}=\frac{2}{9 \sigma_{i}^{2}} \frac{3+R}{4}\left(\frac{\hat{R}_{r} R_{r}^{(\sigma)}}{R_{r}}+\frac{\hat{R}_{X} R_{X}^{(\sigma)}}{R_{X}}\right)\left[3+4 R-2 R^{2}-2(3+R)\left(\frac{\hat{R}_{r} R_{r}^{(\sigma)}}{R_{r}}+\frac{\hat{R}_{X} R_{X}^{(\sigma)}}{R_{X}}\right)\right], \quad \\
S_{\phi}=S_{\phi \phi}=0, S_{\sigma}=-\frac{3+R}{2 \sigma_{i}} \frac{\hat{R}_{r} \hat{R}_{X}}{\hat{R}_{\mathrm{DR}}}\left(\frac{R_{r}^{(\sigma)}}{R_{r}}-\frac{R_{X}^{(\sigma)}}{R_{X}}\right)\left(1-\hat{c}_{\mathrm{v}}\right), \\
S_{\sigma \sigma}=\frac{3+R}{2 \sigma_{i}^{2}} \hat{R}_{r} \hat{R}_{X}\left(\frac{R_{r}^{(\sigma)}}{\hat{R}_{r}}-\frac{R_{X}^{(\sigma)}}{R_{X}}\right) \frac{\left(1-\hat{c}_{\mathrm{v}}\right)}{3}\left[2 R^{2}-4 R-3+\frac{3+R}{\hat{R}_{\mathrm{DR}}}\left(\frac{\hat{R}_{r} R_{r}^{(\sigma)}\left(\hat{c}_{\mathrm{v}}+\hat{R}_{\mathrm{DR}}\right)}{R_{r}}+\frac{\hat{R}_{X} R_{X}^{(\sigma)}\left(1+\hat{R}_{\mathrm{DR}}\right)}{R_{X}}\right)\right] .
\end{gathered}
$$

The meanings of the symbols are as follows: $M_{P}$ is the reduced Planck mass. $V$ is the potential of $\phi$ and $V_{\phi}$ and $V_{\phi \phi}$ are the first and second derivatives of $V$, respectively, evaluated when observable scales exit the horizon. $R \equiv 3 R_{\sigma} /\left(4-R_{\sigma}\right) . R_{i}$ is the ratio of the energy density of a fluid $i$ to the total energy density at the decay of $\sigma$, and $\hat{R}_{i}$ is that at the $e^{ \pm}$annihilation. $R_{i}^{(\sigma)}$ is the ratio of energy density of the fluid $i$ generated by $\sigma$ decay at that time. $\sigma_{i}$ is the amplitude of the oscillation of $\sigma$ when it starts to oscillate. $\hat{c}_{\mathrm{v}} \simeq 0.405$ is the ratio of the energy density of neutrino to that of standard model relativistic particles (photons and neutrinos) after the electron-positron annihilation. The excess of $N_{\text {eff }}$ to the standard value in this scenario is

$$
\Delta N_{\mathrm{eff}}=\frac{3 \hat{R}_{X}}{\hat{c}_{\mathrm{v}} \hat{R}_{r}}
$$

\footnotetext{
${ }^{3}$ There are also terms which arises from the product of three quadratic terms of $\delta \phi_{i}$ in $\zeta$ or $S_{\mathrm{DR}}$, as shown in [3.
} 

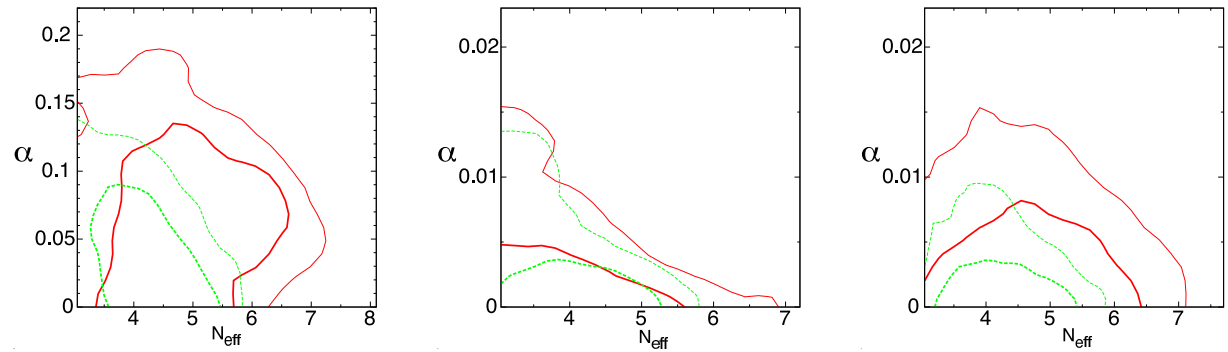

Figure 1: $1 \sigma$ and $2 \sigma$ C.L. constraints in $N_{\text {eff- }} \alpha$ plane from the CMB (red solid) and ALL (green dashed) datasets. From left to right, shown are the constraints for the uncorrelated $(\gamma=0)$, totally correlated $(\gamma=1)$ and totally anti-correlated $(\gamma=-1)$ cases. Note that the scales are not same among three panels.

We refer to Ref. [2] for details and derivations of these quantities and here only comment that $R$ 's in the above equations are written in terms of $r_{\phi}, r_{\sigma}$ and $R_{\sigma}$ and so are (2.12)-2.14).

\section{Constraints from current observations and implications to the model}

In this section we derive constraints on the extra radiation with isocurvature perturbations from current cosmological observations. The energy density of the extra radiation changes the Hubble expansion rate and affect the CMB power spectrum. The free-streaming of DR also affects the CMB perturbation. The isocurvature perturbation of $\mathrm{DR}$, which is equivalent to the neutrino isocurvature perturbation, induce the different type of the CMB spectrum than that induced by the adiabatic perturbation. Due to these effects, we can constrain $N_{\text {eff }}$ and $S_{D R}$ using the CMB data.

We adopt CMB data of WMAP 7-year result [6, 7, 8] and ACT [9, 10, 11] at small angular scales. We also include data from the baryon acoustic oscillation (BAO) in the power spectrum of SDSS galaxies [12] and the direct measurement of the Hubble constant (H0) [13]. Although these observations do not constrain $N_{\text {eff }}$ or $S_{D R}$ directly, including them leads to breaking of the parameter degeneracies. Hereafter, we will refer to sets of combined datasets of WMAP+ACT and $\mathrm{WMAP}+\mathrm{ACT}+\mathrm{BAO}+\mathrm{H} 0$ as "CMB" and "ALL", respectively.

Using a modified version of the publicly available CosmoMC code[14], we derive the constraints on parameters by the MCMC analysis[2]. Figure 1 shows the constraint in $N_{\text {eff }}-\alpha$ plane when $\gamma$ is fixed to 0 or \pm 1 . We see that the data of BAO and $\mathrm{H} 0$ actually tighten the constraints. $N_{\text {eff }}=3.046$ is out of the $1 \sigma$ allowed region in the uncorrelated and totally anti-correlated cases, but consistent with the $2 \sigma$ C.L. level. As is well known, the current CMB data does not show any signatures of the isocurvature perturbation and $\alpha=0$ is consistent in all the cases.

Let us apply the above result to some limiting cases in our cosmological model, which are motivated by particle physics. First, we consider the "uncorrelated" case, where the inflaton does not decay into $X\left(r_{\phi}=1\right)$ and the adiabatic perturbation is dominantly produced by the inflaton $\left(N_{\phi} \gg N_{\sigma}\right)$. Then, the perturbation of $X$ is uncorrelated with $\zeta$. Such a situation can arise in the KSVZ axion model[15, [16], where saxion decays mainly into axion, identifying $\sigma$ with saxion and $\phi$ with another scalar field. Figure 2(a) shows the constraint in the $R_{\sigma}-r_{\sigma}$ plane, in addition to contours of $\alpha$ and $\Delta N_{\text {eff. }}$. Here, $2 R \delta \sigma / \sigma_{i}$ is set to $0.1 \times \zeta_{\phi} \simeq 5 \times 10^{-6}$. 


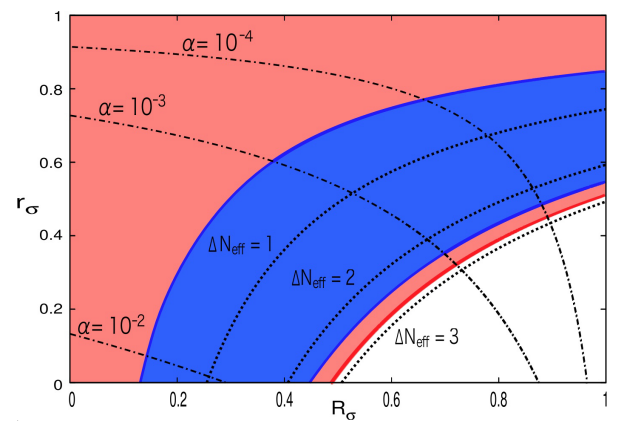

(a) The constraint in $R_{\sigma}-r_{\sigma}$ plane for the uncorrelated case. In this figure $2 R \delta \sigma / \sigma_{i} \simeq 5 \times 10^{-6}$ is fixed.

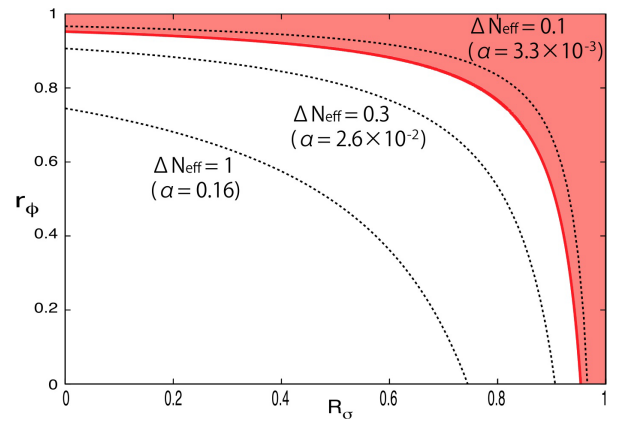

(b) The constraint in $R_{\sigma}-r_{\phi}$ plane for the totally anti-correlated case. There is no $1 \sigma$ allowed region.

Figure 2: The constraints on the model parameters in the uncorrelated case and the totally anti-correlated case. $1 \sigma$ and $2 \sigma$ allowed regions are shown by blue and orange, respectively. Contours of $\alpha$ and $\Delta N_{\text {eff }}$ are also shown. There is the one-to-one correspondence between $\alpha$ and $N_{\text {eff }}$.

Next, we consider the "totally anti-correlated" case, where $\sigma$ does not decay into $X$ ( $r_{\sigma}=$ 1) and it dominantly produces the curvature perturbation $\left(N_{\phi} \ll N_{\sigma}\right)$. $\sigma$ is the curvaton in the original meaning. In this case, $X$ produced by inflaton decay has isocurvature perturbation anticorrelated with the adiabatic one. Such a situation arises in the DFSZ axion model[17, 18], where saxion decay mainly into Higgs bosons, and eventually lighter SM particles. Figure 2(b) shows the constraints on $R_{\sigma}-r_{\phi}$ plane in this case. There is not $1 \sigma$ allowed region and only the $2 \sigma$ allowed region is shown. Besides, we need $R \gtrsim 0.01$, in order not to have too large non-Gaussianity.

\section{Forecast for CMB constraints on the non-Gaussianity parameters}

Although the primordial curvature perturbation is almost Gaussian, current CMB data imply that it might have non-Gaussianity at $1 \sigma$ level 11 and future observations might confirm it. In our cosmological scenario the primordial perturbations have quadratic terms of $\delta \phi_{i}$, which means that they non-Gaussian, like the curvaton scenario. Then bispectra of them might be large and this might lead to the bispectra of the CMB fluctuation detectable in the future. Note that the isocurvature perturbation can be dominant source of non-Gaussianity of the CMB, although it is smaller than the adiabatic one as the source of the power spectra. Like the power spectra, the CMB bispectra induced by $\zeta$ and those by $S_{D R}$ have different feature. Therefore, the CMB bispectra, if detectable, provide us the novel constraints on the extra radiation with the isocurvature perturbation.

The magnitude of each type of bispectra is parametrized by the non-Gaussianity parameters $f_{N L}^{(i)}$. We can perform the Fisher matrix analysis [19, 20, 21] in order to find how strongly they will be constrained in future observations, choosing the fiducial parameter that all $f_{N L}$ 's are 0 [3] . The resultant uncertainties in Planck survey [22] and the cosmic variance limited survey (CVL), in which the instrumental noise is so small that the uncertainties are determined only by the cosmic variance, are summarized in Table 2 for $N_{\text {eff }}=3.04$ and in Table $3 N_{\text {eff }}=4$. If the non-Gaussianity parameters are larger than the values in Table 2 and Table 3 , we will detect the non-Gaussianity in the CMB anisotropy and obtain some information on the extra radiation. 
Table 2: Expected uncertainties for non-Gaussianity parameters for the case with $N_{\text {eff }}=3.04$. "P" and "C" Table 3: Same as in Table 2 but for the case with means Planck and CVL survey respectively. $N_{\text {eff }}=4$.

\begin{tabular}{r|rrrrrr} 
& $f_{\mathrm{NL}}^{(1)}$ & $f_{\mathrm{NL}}^{(2)}$ & $f_{\mathrm{NL}}^{(3)}$ & $f_{\mathrm{NL}}^{(4)}$ & $f_{\mathrm{NL}}^{(5)}$ & $f_{\mathrm{NL}}^{(6)}$ \\
\hline \hline $\mathrm{P}$ & 21 & 126 & 27 & 187 & 257 & 339 \\
\hline $\mathrm{C}$ & 3.5 & 18.3 & 5.0 & 27.2 & 26.4 & 39.3
\end{tabular}

\begin{tabular}{r|rrrrrr} 
& $f_{\mathrm{NL}}^{(1)}$ & $f_{\mathrm{NL}}^{(2)}$ & $f_{\mathrm{NL}}^{(3)}$ & $f_{\mathrm{NL}}^{(4)}$ & $f_{\mathrm{NL}}^{(5)}$ & $f_{\mathrm{NL}}^{(6)}$ \\
\hline \hline $\mathrm{P}$ & 22 & 101 & 21 & 116 & 163 & 164 \\
\hline $\mathrm{C}$ & 3.5 & 14.0 & 3.7 & 15.9 & 15.4 & 17.3
\end{tabular}

\section{Summary}

Motivated by recent observations, in this paper we considered the situation that there are some non-interacting relativistic particles different from neutrinos, dubbed the extra radiation. We focused on the possibility that the dark radiation (DR), the mixture of neutrino and the extra radiation, has the isocurvature perturbation $S_{D R}$, which is motivated by the scenario that the extra radiation is produced by particle decay. We considered the general framework in which there are inflaton $\phi$ and the other light scalar field (curvaton) $\sigma$ and all components in the Universe are produced by their decay. We derived the formulas for the primordial perturbations, the non-Gaussianity parameter for them and the effective neutrino number in terms of the model parameters, the branching ratio of $\phi$ and $\sigma$ decay and the energy density ratio of $\sigma$. Then, we found the constraints on the effective neutrino number $N_{\text {eff }}$ and $S_{D R}$ from current cosmological observations, using the MCMC method, and applied them to some limiting cases in our cosmological model. Finally, we forecast the constraints on non-Gaussianity parameters from future CMB observations using the Fisher analysis.

\section{Acknowledgment}

This work is supported by Grant-in-Aid for Scientific research from the Ministry of Education, Science, Sports, and Culture (MEXT), Japan, No. 14102004 (M.K.), No. 21111006 (M.K. and K.N.), No. 23.10290 (K.M.), No. 22244030 (K.N.) and also by World Premier International Research Center Initiative (WPI Initiative), MEXT, Japan. K.M. and T.S. would like to thank the Japan Society for the Promotion of Science for financial support. The authors acknowledge Kobayashi-Maskawa Institute for the Origin of Particles and the Universe, Nagoya University for providing computing resources useful in conducting the research reported in this paper.

\section{References}

[1] E. Komatsu et al. [WMAP Collaboration], Seven-Year Wilkinson Microwave Anisotropy Probe (WMAP) Observations: Cosmological Interpretation, Astrophys. J. Suppl. 192, 18 (2011) [arXiv:1001.4538 [astro-ph.CO]].

[2] M. Kawasaki, K. Miyamoto, K. Nakayama and T. Sekiguchi, Isocurvature perturbations in extra radiation, JCAP 02 (2012) 022 [arXiv:1107.4962 [astro-ph.CO]].

[3] E. Kawakami, M. Kawasaki, K. Miyamoto, K. Nakayama and T. Sekiguchi, Non-Gaussian isocurvature perturbations in dark radiation, JCAP 07 (2012) 037 [arXiv:1202.4890 [astro-ph.CO]]. 
[4] M. Sasaki, E. D. Stewart, A General analytic formula for the spectral index of the density perturbations produced during inflation, Prog. Theor. Phys. 95, 71, (1996). [astro-ph/9507001].

[5] D. H. Lyth, K. A. Malik, M. Sasaki, A General proof of the conservation of the curvature perturbation, JCAP 05, (2005) 004 [astro-ph/0411220].

[6] B. Gold et al., Seven-Year Wilkinson Microwave Anisotropy Probe (WMAP) Observations: Galactic Foreground Emission, Astrophys. J. Suppl. 192, 15 (2011) [arXiv:1001.4555 [astro-ph.GA]].

[7] D. Larson et al., Seven-Year Wilkinson Microwave Anisotropy Probe (WMAP) Observations: Power Spectra and WMAP-Derived Parameters, Astrophys. J. Suppl. 192, 16 (2011) [arXiv:1001.4635 [astro-ph.CO]].

[8] N. Jarosik et al., Seven-Year Wilkinson Microwave Anisotropy Probe (WMAP) Observations: Sky Maps, Systematic Errors, and Basic Results, Astrophys. J. Suppl. 192, 14 (2011) [arXiv:1001.4744 [astro-ph.CO]].

[9] A. Hajian et al., The Atacama Cosmology Telescope: Calibration with WMAP Using Cross-Correlations, arXiv:1009.0777 [astro-ph.CO].

[10] S. Das et al., The Atacama Cosmology Telescope: A Measurement of the Cosmic Microwave Background Power Spectrum at 148 and 218 GHz from the 2008 Southern Survey, Astrophys. J. 729, 62 (2011) [arXiv:1009.0847 [astro-ph.CO]].

[11] J. Dunkley, R. Hlozek, J. Sievers et al., The Atacama Cosmology Telescope: Cosmological Parameters from the 2008 Power Spectra, Astrophys. J. 739, 52 (2011) [arXiv:1009.0866 [astro-ph.CO]].

[12] B. A. Reid et al. [SDSS Collaboration], Baryon Acoustic Oscillations in the Sloan Digital Sky Survey Data Release 7 Galaxy Sample, Mon. Not. Roy. Astron. Soc. 401, 2148 (2010) [arXiv:0907.1660 [astro-ph.CO]].

[13] A. G. Riess et al., A Redetermination of the Hubble Constant with the Hubble Space Telescope from a Differential Distance Ladder, Astrophys. J. 699, 539 (2009) [arXiv:0905.0695 [astro-ph.CO]].

[14] A. Lewis, S. Bridle, Cosmological parameters from CMB and other data: A Monte Carlo approach, Phys. Rev. D66, 103511 (2002). [astro-ph/0205436].

[15] J. E. Kim, Weak Interaction Singlet and Strong CP Invariance, Phys. Rev. Lett. 43, 103 (1979);

[16] M. A. Shifman, A. I. Vainshtein, V. I. Zakharov, Can Confinement Ensure Natural CP Invariance of Strong Interactions?, Nucl. Phys. B166, 493 (1980).

[17] M. Dine, W. Fischler, M. Srednicki, A Simple Solution to the Strong CP Problem with a Harmless Axion, Phys. Lett. B104, 199 (1981);

[18] A. R. Zhitnitsky, On Possible Suppression Of The Axion Hadron Interactions., Sov. J. Nucl. Phys. 31, 260 (1980) [Yad. Fiz. 31, 497 (1980)].

[19] E. Komatsu and D. N. Spergel, Acoustic signatures in the primary microwave background bispectrum, Phys. Rev. D 63, 063002 (2001) [arXiv:astro-ph/0005036].

[20] D. Babich and M. Zaldarriaga, Primordial bispectrum information from CMB polarization, Phys. Rev. D 70, 083005 (2004) [arXiv:astro-ph/0408455].

[21] A. P. S. Yadav, E. Komatsu and B. D. Wandelt, Fast Estimator of Primordial Non-Gaussianity from Temperature and Polarization Anisotropies in the Cosmic Microwave Background, Astrophys. J. 664, 680 (2007) [arXiv:astro-ph/0701921].

[22] Planck Collaboration, The Scientific programme of planck, astro-ph/0604069. 